\title{
Immature Leukocyte to Leukocytes Ratio Measurement
}

National Cancer Institute

\section{Source}

National Cancer Institute. Immature Leukocyte to Leukocytes Ratio Measurement. NCI Thesaurus. Code 127626.

The determination of the ratio of immature leukocytes compared to total leukocytes present in a sample. The measurement may be expressed as a ratio or percentage. 